\title{
Detection of mSiglec-E, in solution and expressed on the surface of Chinese hamster ovary cells, using sialic acid functionalised gold nanoparticles $^{\dagger}$
}

Claire L. Schofield, ${ }^{a}$ María J. Marín, ${ }^{a}$ Martin Rejzek, ${ }^{b}$ Paul R. Crocker, ${ }^{c}$ Robert A. Field ${ }^{b^{*}}$ and David A. Russell ${ }^{a^{*}}$

${ }^{a}$ School of Chemistry, University of East Anglia, Norwich Research Park, Norwich, NR4 7TJ, UK.E-mail:d.russell@uea.ac.uk

${ }^{b}$ Department of Biological Chemistry, John Innes Centre, Norwich Research Park, Norwich, NR4 7UH, UK. E-mail: rob.field@jic.ac.uk

'Division of Cell Signalling and Immunology, Wellcome Trust Biocentre, College of Life Sciences, University of Dundee, Dundee, DD1 5EH, UK

${ }^{\dagger}$ Electronic Supplementary Information (ESI) available. See DOI: XXX 


\begin{abstract}
Sialic acids are widespread in biology, fulfilling a wide range of functions. Their cognate lectin receptors - Siglecs - are equally diverse and widely distributed, with different Siglecs found within distinct populations of cells in the haemopoietic, immune and nervous systems. A convenient way to assay ligand recognition of soluble Siglecs would be useful, as would methods for the concomitant assessment of Siglec distribution on cell surfaces. Here we report the use of gold nanoparticles functionalised with a sialic acid ligand diluted with a polyethylene glycol (PEG) ligand for the plasmonic detection of a soluble form of murine Siglec-E (mSiglec-E-Fc fusion protein) and, importantly, for the specific detection of the same siglec expressed on the surface of mammalian cells. These sialic acid functionalised nanoparticles are shown to overcome problems such as cellular cis interactions and low Siglec-ligand affinity. The gold nanoparticles were functionalised with various ratios of sialic acid:PEG ligands and the optimum ratio for the detection of murine Siglec-E was established based on the plasmonic detection of the soluble pre-complexed recombinant form of murine Siglec-E (mSiglec-E-Fc fusion protein). The optimum ratio for the detection of the fusion protein was found to be sialic acid:PEG ligands in a 50:50 ratio (glyconanoparticles 1 ). The optimised glyconanoparticles 1 were used to recognise and bind to the murine Siglec-E expressed on the surface of transfected Chinese hamster ovary cells as determined by transmission electron microscopy.
\end{abstract}




\section{Introduction}

Siglecs are a family of sialic acid-binding immunoglobulin-like lectins that are found on many immune system cell types where they regulate the functions and interactions of such cells. ${ }^{1-}$ 4 Siglecs are type-I cell surface transmembrane receptors that are formed by 2-17 extracellular immunoglobulin-like domains, ${ }^{4}$ including varying numbers of $\mathrm{C} 2$-set domains (from 16 in Siglec-1 to 1 in Siglec-3) and an amino-terminal V-set that contains a conserved arginine residue that mediates the carbohydrate binding activity. ${ }^{5}$ Human Siglecs (hSiglecs) and murine Siglecs (mSiglecs) can be divided into two sub-groups based on sequence similarity and evolutionary conservation. ${ }^{6}$ The first group consists of sialoadhesin (Siglec-1), CD22 (Siglec-2), myelin-associated glycoprotein (Siglec-4) and Siglec-15. The second subgroup, known as the CD33-related Siglecs, consists of CD33 (Siglec-3) plus Siglecs-5-11 and 14 in humans and Siglec-E-H in mice; which are found mainly on mature cells of the immune system, such as natural killer (NK) cells, mast cells, neutrophils, eosinophils and macrophages. CD33-related Siglecs are involved in the inhibition of proliferation and in the induction of apoptosis, ${ }^{7,8}$ inhibit the killing function of NK cells against tumours expressing sialylated glycans, ${ }^{9}$ and are highly expressed in acute myeloid leukaemia and human blood leukocytes. ${ }^{10,} 11$ In mouse, neutrophils and tissues rich in leukocytes express high levels of mSiglec-E. mSiglec-E is structurally similar to both hSiglec-7 and -9 , with all three siglecs containing an amino-termina V-set and two C2-set immunoglobulin domains in the extracellular membrane and an immunoreceptor tyrosine-based inhibitory (ITIM) motif and an ITIM-like domain in the cytoplasmic tail (Fig. S1). ${ }^{3}$ Thus, mSiglec-E is expressed in most of the cell types that express either hSiglec-7 or hSiglec-9. ${ }^{12}$ However, although these human and mice Siglecs are structurally similar, they differ in some of the amino acid residues present in each of the structural components ${ }^{13}$ (the sequence similarity between hSiglec-7 and -9 and $\mathrm{mSiglec}-\mathrm{E}$ is $52 \%$ and $53 \%$, respectively). ${ }^{14}$ The peptide variability between these three Siglecs is responsible for their differences in sialic acid binding specificities. ${ }^{13}$ To date, there is insufficient information to fully understand the biological roles that hSiglecs play in vivo. The development of murine-based animal models and the study of the structurally equivalent hSiglecs in mice have provided some insights. ${ }^{15}$ 
CD33-related Siglecs have been exploited as targets for different treatment strategies. ${ }^{16}$ For example, CD33 and CD22 are markers of myeloid leukemia and B-cell malignancies and have been used as targets of antibody-drug conjugate-based treatments. ${ }^{17}$ An immunoconjugate of anti-CD33-antibody with the antibiotic calicheamicin was commercialised for the treatment of acute myeloid leukaemia (Mylotarg ${ }^{\circledR}$ ). ${ }^{18}$ hSiglec-7, overexpressed on a variety of cancer cells, ${ }^{19}$ has been used as a target for the drug delivery of antibody-functionalised poly(lactide-co-glycolide) nanoparticles. ${ }^{20}$ Recently, the use of liposomal nanoparticles functionalised with glycan ligands that bind specifically to a Siglec of interest such as hCD33 and hCD $22,{ }^{21}$ sialoadhesin/Siglec- $1,{ }^{22}$ Siglec- $9^{23}$ and Siglec- $-7^{24}$ has been extensively reported by Paulson and co-workers. These studies use fluorescence-based techniques to elucidate the behaviour of the nanoparticles within the cells. However, such techniques do not allow single particle detection and cannot provide information regarding localisation at the structural level that could be achieved with electron microscopy. Recently, artificial viruslike glycosphingolipid-functionalised nanoparticles incorporating $80 \mathrm{~nm}$ gold nanoparticles were used, in combination with scanning electron microscopy, to understand the behaviour of human immunodeficiency virus-1 when bound to Siglec-1 receptors expressed on HeLa cells. $^{25}$

Gold nanoparticles (AuNPs) allow for the use of electron microscopy techniques to obtain high resolution images, and are ideal for the development of plasmonic bioassays since the colour of a solution of nanoparticles depends on the localised surface plasmon fields which are, in turn, dependent on the distance between particles. ${ }^{26}$ AuNPs have been functionalised with carbohydrate derivatives (glyconanoparticles) and used for the plasmonic detection of analytes of biological interest. ${ }^{27}$ AuNPs ( $c a .15 \mathrm{~nm}$ ) exhibit a surface plasmon absorption band centred at $520 \mathrm{~nm}$ and, consequently, are red in colour. When the particles aggregate due to the presence of the target analyte, the surface plasmon absorption band red-shifts producing a colour change in the solution. With this change of colour, glyconanoparticles have been used for the detection of biological targets such as concanavalin A (ConA), ${ }^{28,} 29$ cholera toxin ${ }^{30}$ and Ricinus communis Agglutinin 120 (RCA 120$){ }^{31}$ Sialic acid functionalised gold nanoparticles have been used for the inhibition and detection of influenza virus ${ }^{32,33}$ and more recently for the detection and discrimination between human and avian influenza viruses. ${ }^{34}$ Silver nanoparticles functionalised with both sialic acid 
and galactose derivatives have been used in combination with extinction and surface enhanced Raman (SER) spectroscopies for the detection of cholera toxin. ${ }^{35}$

A further advantage that the glyconanoparticles possess for the detection of Siglecs expressed on the surface of cells is their potential to overcome problems that other detection methods encounter including the presence of cis interactions and the low Siglecligand affinity. When interactions between Siglec and sialic acid are described, the initial assumption is that the Siglec of one cell binds glycans terminating in sialic acid that are on a second cell (in trans) and therefore, an intercellular adhesion takes place. ${ }^{36-38}$ However, since the concentration of sialic acid terminated glycans on the surface of a cell is high, Siglecs can also bind those sialic acids of glycans expressed on the same cell (in cis). ${ }^{36-38}$ The binding sites of the Siglecs are then 'masked' by the sialic acid in cis, which can be reduced if an external ligand with higher affinity towards the Siglec is present. However, Siglec-ligand interactions are usually weak. These problems can be overcome using multivalent ligandbased probes $^{36}$ such as glyconanoparticles where a large number of carbohydrate-based ligands can be attached onto the surface of AuNPs.

In this paper we describe, to the best of our knowledge, the first glyconanoparticles that enable the plasmonic detection of Siglecs in solution and, importantly, using the same glyconanoparticle based platform for the detection of Siglecs expressed on the surface of transfected Chinese hamster ovary $(\mathrm{CHO})$ cells using transmission electron microscopy (TEM). AuNPs (ca. $15 \mathrm{~nm}$ ) were functionalised with a S-linked sialic acid derivative ligand (sialic acid ligand 1 ) and a thiolated polyethylene glycol derivative ligand (PEG ligand 2) yielding glyconanoparticles (Fig. 1). Different ratios of the sialic acid ligand 1 and PEG ligand 2 were studied and the optimum ratio was determined following the plasmonic detection of mSiglec-E-Fc cross-linked with an anti-Fc-IgG-antibody (mSiglec-E-Fc/antibody-complex) in solution (Fig. 2a). The optimum ratio for the plasmonic detection of the pre-complexed mSiglec-E-Fc was found to be 50:50 (glyconanoparticles 1 ). This solution based plasmonic assay enabled the study of selectivity and specificity of the designed platforms in an easy and rapid manner. The optimised glyconanoparticles 1 were subsequently used for the detection of mSiglec-E expressed on the surface of transfected $\mathrm{CHO}$ cells. The sialic acid 
ligand on the glyconanoparticles recognised and interacted with the mSiglec-E expressed on the cell surface (Fig. 2b) which was then visualised using TEM.

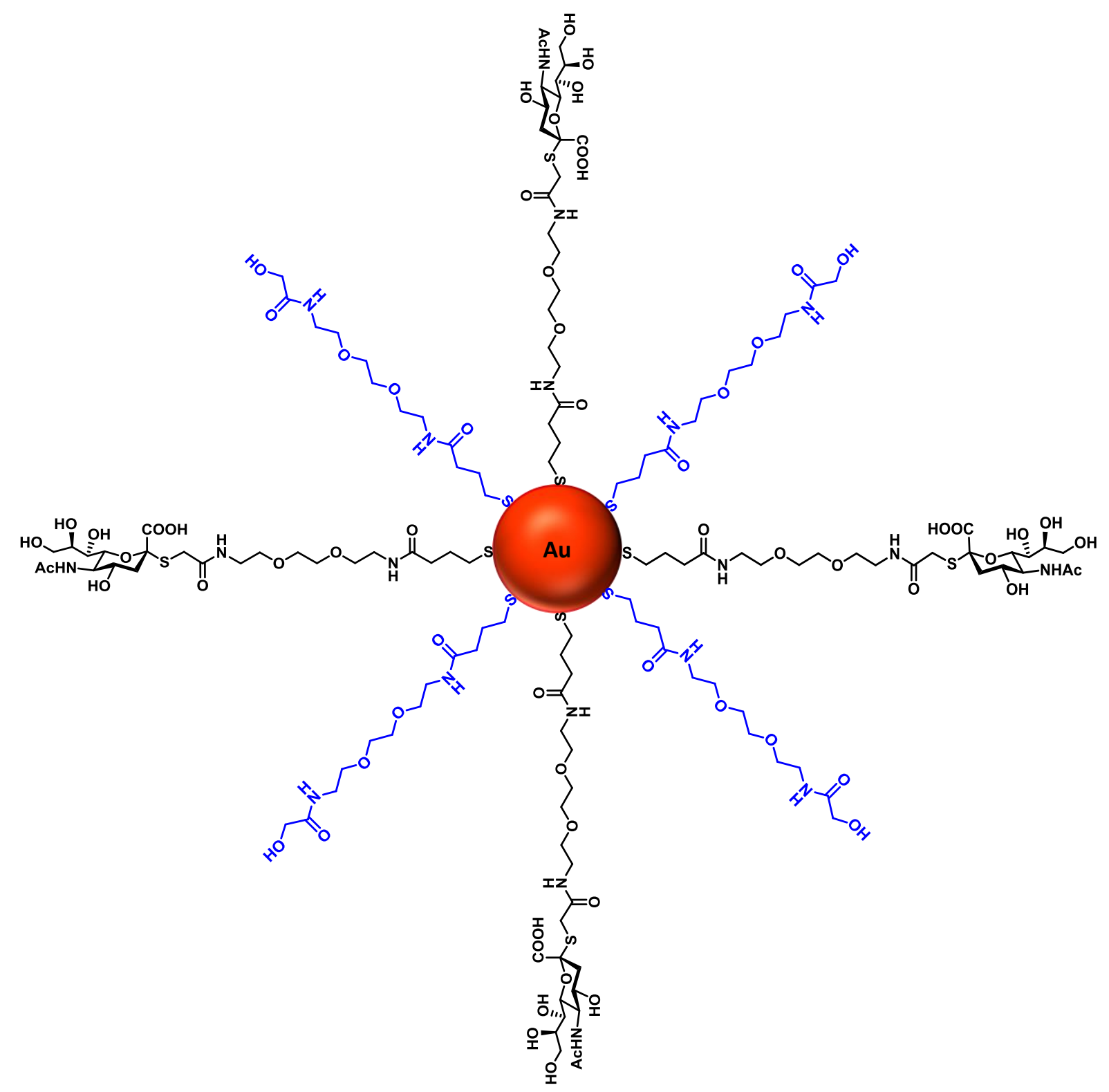

Fig. 1. Schematic representation of the sialic acid ligand $\mathbf{1}$ (black):PEG ligand $\mathbf{2}$ (blue) functionalised gold nanoparticles. 
a

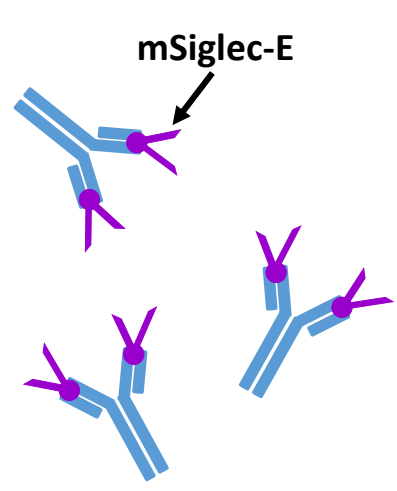

mSiglec-E cross-

linked with anti-FcIgG antibody

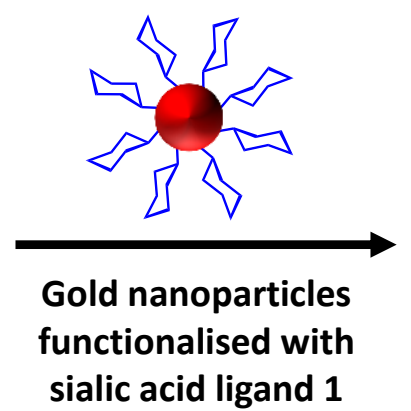

sialic acid ligand 1

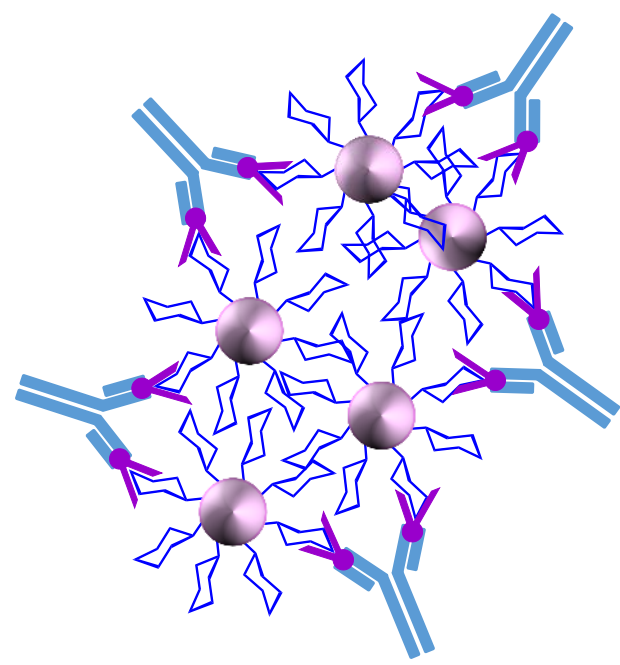

b
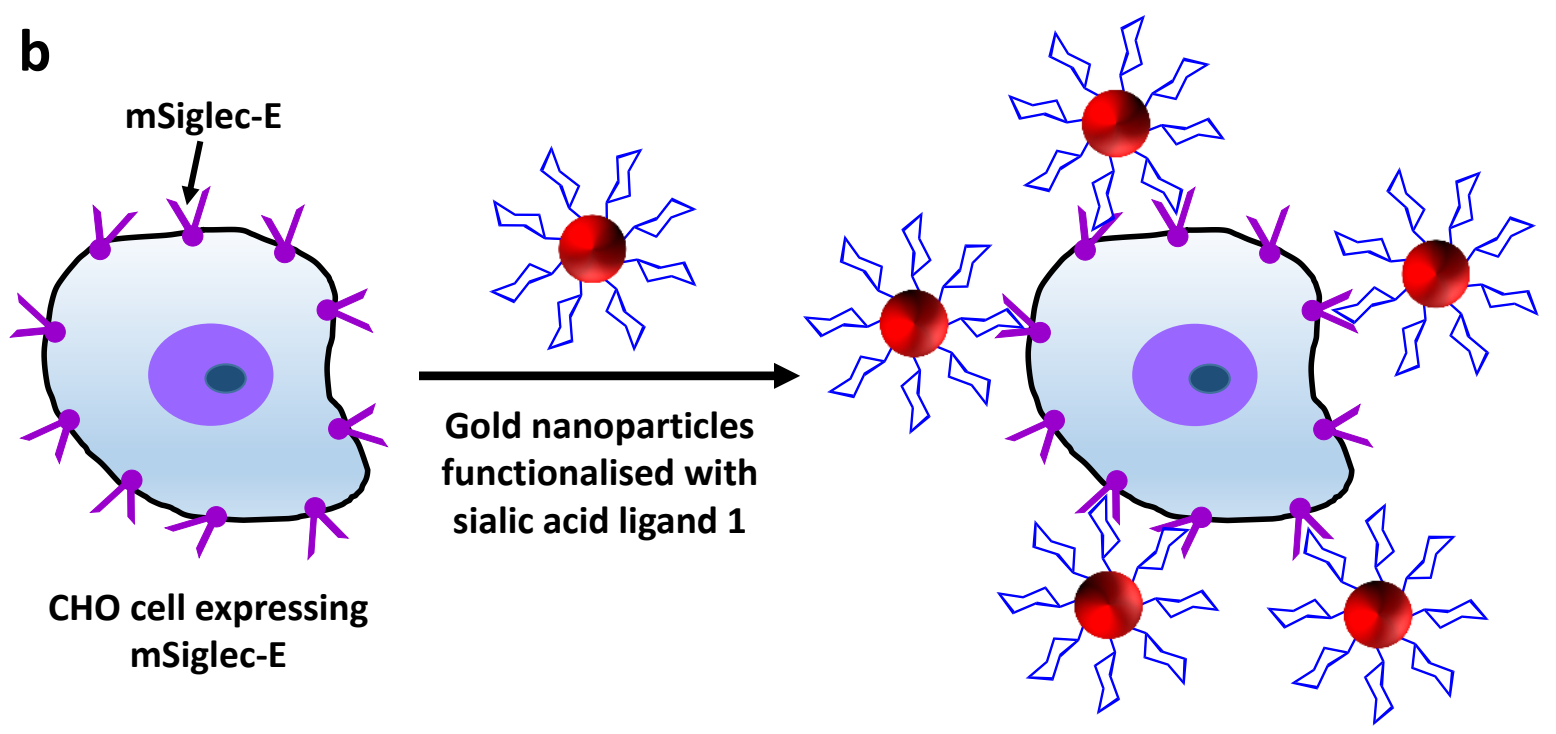

Gold nanoparticles functionalised with sialic acid ligand 1

\section{$\mathrm{CHO}$ cell expressing mSiglec-E}

Fig. 2. Schematic representation of the binding of the sialic acid ligand 1 on the glyconanoparticles to: a) the mSiglec-E cross-linked with an anti-Fc-IgG antibody inducing the aggregation of the glyconanoparticles; and $\mathbf{b}$ ) the mSiglec-E expressed on the surface of transfected $\mathrm{CHO}$ cells. Nanoparticles and cells not to scale.

\section{Experimental}

\section{Synthesis of the sialic acid ligand 1}

Sialic acid ligand 1 was synthesised using a previously reported protocol ${ }^{34}$ as detailed in Scheme 1 and in the Electronic Supplementary Information. 


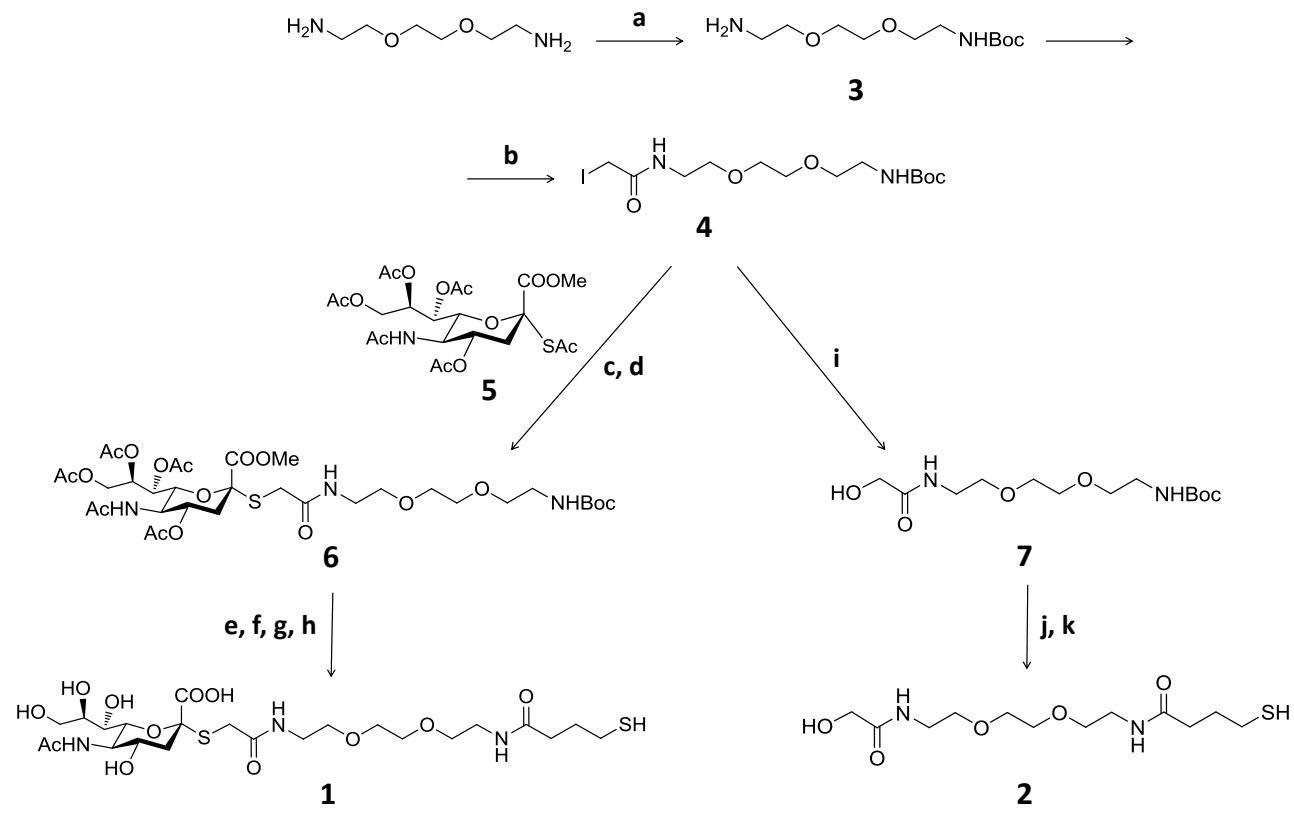

Scheme 1 Synthesis of the sialic acid ligand 1 and PEG ligand 2. a) (Boc) ${ }_{2} \mathrm{O}, \mathrm{DCM}$, from $0{ }^{\circ} \mathrm{C}$ to r.t.; b) iodoacetic anhydride, $\mathrm{Et}_{2} \mathrm{O}$, r.t.; c) 1 . $\mathrm{NaOMe} / \mathrm{MeOH},-40{ }^{\circ} \mathrm{C}$ and 2. Amberlite IR$120\left(\mathrm{H}^{+}\right),-40^{\circ} \mathrm{C}$; d) 5, DIPEA, DCM; e) $\mathrm{NaOMe} / \mathrm{MeOH}$, r.t.; f) $1 \mathrm{M} \mathrm{NaOH}$, r.t.; g) TFA, DCM, r.t.; h) $\gamma$-thiobutyrolactone, aq. $\mathrm{NaHCO}_{3} / \mathrm{EtOH}, \mathrm{DTT}, 50{ }^{\circ} \mathrm{C}$; i) $\mathrm{K}_{2} \mathrm{CO}_{3}, \mathrm{DMF}: \mathrm{H}_{2} \mathrm{O}(1: 1), 80{ }^{\circ} \mathrm{C}$; j) TFA, DCM; and k) $\gamma$-thiobutyrolactone, $0.5 \mathrm{M} \mathrm{NaHCO}_{3}$, EtOH, DTT, $50^{\circ} \mathrm{C}$.

\section{Synthesis of the PEG ligand 2}

PEG ligand 2 was synthesised following the protocol summarised in Scheme 1 which includes the following steps:

Synthesis of N-Boc-2,2'-(ethylenedioxy)bis(ethylamine) (3)

Compound $\mathbf{3}$ was synthesised from the corresponding diamine following a published procedure. $^{39}$

Synthesis of t-butyl 2-(2-(2-(2-iodoacetamido)ethoxy)ethoxy)ethylcarbamate (4) lodoacetic anhydride (555 mg, $1.57 \mathrm{mmol})$ was dissolved in absolute diethylether $\left(\mathrm{Et}_{2} \mathrm{O}\right.$, $10 \mathrm{~mL}$ ) and the solution was added to a stirred solution of compound 3 (299.6 mg, $1.21 \mathrm{mmol})$ in absolute diethylether $(10 \mathrm{~mL})$ under $\mathrm{N}_{2}$ and with the exclusion of light. The mixture was allowed to stir for $60 \mathrm{~min}$ at r.t. and the formation of product was followed by 
$\operatorname{TLC}\left(R_{f}=0.67\right.$, ethyl acetate/methanol 20:1). The volatiles were evaporated in vacuo and the residue was subjected to column chromatography on silica gel (using first ethyl acetate followed by ethyl acetate/methanol $20: 1$ ) to give pure 4 (353.5 $\mathrm{mg}, 70 \%$ ) as a pale yellow oil. The characterisation of compound $\mathbf{4}$ is given in the Electronic Supplementary Information.

Synthesis of N-(2-(2-(2-(2-hydroxyacetamido)ethoxy)ethoxy)ethyl)-4-mercaptobutanamide (2)

$4(50 \mathrm{mg}, 120 \mu \mathrm{mol})$ and $\mathrm{K}_{2} \mathrm{CO}_{3}(83 \mathrm{mg}, 600 \mu \mathrm{mol})$ were heated to $80{ }^{\circ} \mathrm{C}$ in a mixture of dimethylformamide (DMF, $0.5 \mathrm{~mL}$ ) and water $(0.5 \mathrm{~mL})$. After $6 \mathrm{~h}$, when TLC showed complete conversion (hydroxyacetamide 7; $R_{f}=0.42$, chloroform/methanol 10:1), the volatiles were evaporated in vacuo. Acetic acid $(50 \%, 234 \mu \mathrm{L})$ was added and the volatiles

were evaporated in vacuo. The crude t-butyl 2-(2-(2-(2hydroxyacetamido)ethoxy)ethoxy)ethylcarbamate (7) was dissolved in dichloromethane (DCM, $1 \mathrm{~mL}$ ) and trifluoroacetic acid (TFA, $1 \mathrm{~mL}$ ) was added. The mixture was stirred at r.t. for $3 \mathrm{~h}$ and then the volatiles were evaporated. The residue was dissolved in a mixture of sodium bicarbonate $\left(\mathrm{NaHCO}_{3}, 0.5 \mathrm{M}, 2.76 \mathrm{~mL}\right.$ ) and ethanol (EtOH, $2.20 \mathrm{~mL}$ ) and dithiothreitol (DTT, $46.3 \mathrm{mg}, 300.3 \mu \mathrm{mol})$ and $\gamma$-thiobutyrolactone $(52 \mu \mathrm{L}, 600.5 \mu \mathrm{mol})$ were added. The mixture was stirred overnight at $50{ }^{\circ} \mathrm{C}$ under $\mathrm{N}_{2}$. Using $0.5 \mathrm{M} \mathrm{HCl}$ the $\mathrm{pH}$ of the mixture was adjusted to 6.0. Volatiles were evaporated in vacuo and the wet residue was freeze-dried. The solid was taken into methanol $(\mathrm{MeOH}, 5 \mathrm{~mL})$ and solids were filtered off. The residue was subjected to column chromatography on silica gel $(6 \mathrm{~g}$, chloroform/methanol, stepwise gradient 30:1, 20:1, 10:1, 5:1) to give pure PEG ligand 2 ( $35.3 \mathrm{mg}, 95 \%$ over 3 steps). The characterisation of compound $\mathbf{2}$ is given in the Electronic Supplementary Information.

Synthesis of gold nanoparticles and functionalisation with sialic acid ligand 1 and PEG ligand 2 - glyconanoparticles

Water soluble citrate-reduced gold nanoparticles were prepared via the citrate reduction of hydrogen tetrachloroaurate $\left(\mathrm{HAuCl}_{4}\right)$ following the method reported by Enüstün and Turkevich. ${ }^{40}$ Briefly, aqueous solutions of $\mathrm{HAuCl}_{4} \cdot 3 \mathrm{H}_{2} \mathrm{O}(12.5 \mathrm{mg}, 32 \mu \mathrm{mol}$, in $100 \mathrm{~mL})$ and sodium citrate tribasic dihydrate $(50 \mathrm{mg}, 168 \mu \mathrm{mol}$, in $50 \mathrm{~mL}$ ) were prepared and heated to 
$60{ }^{\circ} \mathrm{C}$. The sodium citrate solution was rapidly added to the gold solution while stirring vigorously. The temperature was increased to $85^{\circ} \mathrm{C}$ and the solution was stirred for $2 \mathrm{~h}$. A clear red gold nanoparticle solution was obtained that was cooled to room temperature and filtered through a Miller GP syringe driven filter unit $(0.22 \mu \mathrm{m})$. The concentration of the citrate stabilised gold nanoparticles solution was approximately $3 \mathrm{nM}$.

Varying molar ratios of sialic acid ligand 1 and PEG ligand 2 (100:0, 50:50, 25:75, 5:95 and 0:100) were separately added to aliquots of the freshly prepared $3 \mathrm{nM}$ AuNPs (17 $\mathrm{mL}$ ) and stirred for $48 \mathrm{~h}$ at r.t. to ensure self-assembly of the ligands onto the gold surface. The total concentration of thiol ligands in the solution was $c a .3 \mathrm{nmol} / \mathrm{mL}$. The samples were centrifuged for $25 \mathrm{~min}$ at $23,710 \mathrm{xg}$ to remove the excess ligands. The nanoparticles were resuspended in Tris-buffer (10 mM, pH 7.6). The centrifugation process was repeated a total of three times.

\section{Cross-linking of mSiglec-E and hSiglec-7 with goat anti-human (Fc specific) antibody (mSiglec-E-Fc/antibody-complex and hSiglec-7-Fc/antibody-complex, respectively)}

The Fc fusion proteins mSiglec-E and hSiglec-7 were cross-linked with an anti-Fc antibody, goat anti-human-Fc-IgG antibody, prior to use following a previously reported protocol. ${ }^{41}$ Briefly, a $50 \mu \mathrm{L}$ mixture of the Fc fusion protein $(2 \mathrm{mg} / \mathrm{mL})$ and goat anti-human-Fc-lgG antibody $(2 \mathrm{mg} / \mathrm{mL})$ was incubated for $1 \mathrm{~h}$ at room temperature. The resulting complex was diluted in phosphate buffer saline $(1.6 \mathrm{~mL})$ prior to use. The complexes obtained were denoted as: mSiglec-E-Fc/antibody-complex for the mSiglec-E-Fc cross-linked with the antibody and hSiglec-7-Fc/antibody-complex for the hSiglec-7-Fc cross-linked with the antibody.

Plasmonic detection of $\mathrm{mSiglec-E-Fc/antibody-complex} \mathrm{using} \mathrm{the} \mathrm{varying} \mathrm{ratio} \mathrm{sialic} \mathrm{acid}$ ligand 1:PEG ligand 2 functionalised gold nanoparticles

Increasing amounts of mSiglec-E-Fc/antibody-complex (from 0 to $4.7 \mu \mathrm{g}$ ) were added to a sample of the sialic acid ligand 1:PEG ligand 2 functionalised AuNPs of varying ratios (concentration of nanoparticles $=c a .2 \mathrm{nM}$ ). The UV-Vis extinction spectrum of each glyconanoparticle solution was measured before and after addition of the corresponding amount of mSiglec-E-Fc/antibody-complex. 


\section{Control experiments}

Plasmonic detection of anti-Fc antibody

Glyconanoparticles 1 ( $c a .2 \mathrm{nM}$ ) were reacted with goat anti-human-Fc-IgG antibody (from 0 to $6.25 \mu \mathrm{g}$ ). The UV-Vis extinction spectrum of the solution was measured before and after addition of increasing amounts of antibody.

Plasmonic detection of mSiglec-E using gold nanoparticles functionalised only with PEG ligand 2

mSiglec-E-Fc/antibody-complex $(2.35 \mu \mathrm{g})$ was added to a sample of PEG ligand 2 functionalised gold nanoparticles (ca. $2 \mathrm{nM}$ ). The UV-Vis extinction spectrum of the solution was measured before and after addition of the mSiglec-E-Fc/antibody-complex.

\section{Plasmonic detection of hSiglec-7-Fc/antibody-complex}

hSiglec-7-Fc/antibody-complex $(2.35 \mu \mathrm{g})$ was added to a sample of glyconanoparticles $\mathbf{1}$ (ca. $2 \mathrm{nM}$ ). The UV-Vis extinction spectrum of the solution was measured before and after addition of the hSiglec-7-Fc/antibody-complex.

\section{Cellular experiments using $\mathrm{CHO}$ cells expressing mSiglec-E and wild-type $\mathrm{CHO}$}

$\mathrm{CHO}$ cells were transfected to express mSiglec-E as described previously. ${ }^{42}$ Both wild-type CHO cells and $\mathrm{CHO}$ cells expressing mSiglec-E were cultured in HAMS F12 medium supplemented with $10 \%$ foetal calf serum, penicillin $(100 \mathrm{U} / \mathrm{mL})$ and streptomycin $(100 \mu \mathrm{g} / \mathrm{mL})$.

To perform the cellular experiments, glyconanoparticles 1 (ca. $2 \mathrm{nM}$ ) were mixed with $10^{6}$ cells, either $\mathrm{CHO}$ cells expressing mSiglec-E or with wild-type $\mathrm{CHO}$ cells, for $10 \mathrm{~min}$ at r.t.

\section{Transmission electron microscopy imaging}

TEM imaging of glyconanoparticles in the absence and presence of mSiglec-E-Fc/antibodycomplex

A sample of the glyconanoparticles $(c a .10 \mu \mathrm{L})$, before and after being reacted with mSiglecE-Fc/antibody-complex $(2.35 \mu \mathrm{g})$ for $2 \mathrm{~h}$, was deposited on a holey carbon film 300 mesh 
copper grids from Agar Scientific (UK) and imaged using a Jeol 200EX transmission electron microscope.

\section{TEM imaging of cells}

All of the cellular samples studied in this work, $\mathrm{CHO}$ cells expressing mSiglec-E incubated and non-incubated with glyconanoparticles 1 and wild-type $\mathrm{CHO}$ cells incubated with glyconanoparticles 1, were treated prior to TEM imaging using a similar protocol. The cell suspension in a screw cap Eppendorf tube was fixed in $5 \%(\mathrm{v} / \mathrm{v})$ glutaraldehyde and left at $4{ }^{\circ} \mathrm{C}$ for 30-60 min. The sample was vortexed and then spun in a bench-top centrifuge at 10,000 rpm for $10 \mathrm{~min}$. The pellet was washed in phosphate buffer (pH 7.2, ca. $1 \mathrm{~mL}$ ) and then resuspended. Following a second centrifugation at 10,000 rpm for $10 \mathrm{~min}$, the pellet was dehydrated through a graded ethanol series of 30,50, 70, 90, 98 and 100\% (aq.) ethanol $(1 \mathrm{~mL}), c a .15 \mathrm{~min}$ in each solution. From $100 \%$ ethanol, $0.5 \mathrm{~mL}$ of the solution was replaced by LR White resin medium grade (Agar Scientific, $0.5 \mathrm{~mL}$ ). The mixture was left to rest for $1 \mathrm{~h}$ at room temperature. The solution was then removed and replaced with $100 \%$ resin. The sample was vortexed to resuspend the pellet (cells) and left overnight (between 12 and $18 \mathrm{~h}$ ) at room temperature. The sample was centrifuged at 10,000 rpm for $10 \mathrm{~min}$ and the resin was removed. The Eppendorf tube was filled to the brim with resin that sets under anaerobic conditions and the sample was polymerised overnight in an oven at $60{ }^{\circ} \mathrm{C}$. The polymerised (hardened) resin was sectioned on an LKB Nova ultramicrotome. Using glass knives, sections at 70-100 nm were taken of the pellet and placed on a copper 100 mesh grid coated with a Parlodion film. For stained samples, the sections were stained with uranyl acetate (1\% aq.) and $2 \%$ lead citrate for $10 \mathrm{~min}$. The samples were viewed using a Jeol 200EX transmission electron microscope operated at $80 \mathrm{kV}$.

\section{Results and discussion}

\section{Synthesis and characterisation of sialic acid ligand 1 and PEG ligand 2}

The synthesis of the sialic acid ligand $\mathbf{1}$ and the PEG ligand $\mathbf{2}$ was achieved as shown in Scheme 1. The PEG ligand $\mathbf{2}$ and part of the aliphatic side chain of the sialic acid ligand $\mathbf{1}$ were synthesised starting from $\mathrm{N}$-Boc-2,2'-(ethylenedioxy)bis(ethylamine) (3) prepared from the corresponding diamine following a published procedure. ${ }^{39} \mathbf{3}$ was reacted with iodoacetic 
anhydride to give the corresponding iodoacetamide 4 in $70 \%$ yield. Compound 4 was subjected to a base catalysed hydrolysis of the iodide in wet dimethylformamide ${ }^{43}$ to give the corresponding hydroxyacetamide 7. Boc deprotection in compound $\mathbf{7}$ was undertaken followed by conversion of the resulting free amine to 4-thiobutyroamide 2 using $\gamma$ thiobutyrolactone in a mixture of $0.5 \mathrm{M} \mathrm{NaHCO}_{3}$ buffer $(\mathrm{pH} c a .9 .0)$ and ethanol under reducing conditions provided by the presence of dithiothreitol ${ }^{44}$ to give the PEG ligand $\mathbf{2}$ in 95\% yield over 3 steps. For the synthesis of the sialic acid ligand 1, compound 4 was first alkylated with an $\alpha$-configured sialic acid thioacetate followed by a deprotection and reaction of the free amine with $\psi$-thiobutyrolactone. Acidification of the formed sodium salt of the ligand afforded the desired sialic acid ligand $\mathbf{1}$.

\section{Synthesis of the glyconanoparticles}

The synthesis of water soluble AuNPs (ca. $15 \mathrm{~nm}$ ) was achieved using citrate as both the reducing agent and the stabiliser of the gold core. ${ }^{40}$ The suspension of the citrate AuNPs was red in colour as a consequence of the surface plasmon absorption band centred at ca. $520 \mathrm{~nm}$. Citrate-reduced AuNPs were functionalised with varying ratios of the sialic acid ligand 1:PEG ligand 2 (100:0, 50:50, 25:75 and 5:95) to establish whether the binding of the mSiglec-E to the sialic acid ligand $\mathbf{1}$ on the glyconanoparticles was ligand density dependent. All of the synthesised glyconanoparticles yielded a deep red aqueous suspension that had an average size of $c a .15 \mathrm{~nm}$ (a histogram showing the size distribution of the glyconanoparticles 1 can be seen in Fig. S2, with an average size of $14.9 \pm 1.7 \mathrm{~nm}$ ).

\section{Plasmonic detection of Siglecs in solution using the glyconanoparticles}

Siglecs are naturally expressed on the surface of cells where they can occur in clusters via lateral diffusion. ${ }^{3}$ To mimic the cell surface configuration of these proteins, a multivalent presentation of the Siglecs is required. Surface plasmon resonance analysis has previously shown that, without cross-linking with an antibody, monomeric hSiglec-7-Fc exhibits only a weak binding to sialic acid derivatives. ${ }^{41}$ Multivalent formats for the study of selective recognition by Siglecs have been also achieved following pre-complexation of mSiglecs-Fc and hSiglecs-Fc with the corresponding antibodies. ${ }^{45-47}$ The need of the formation of a precomplex Siglec-Fc-antibody to observe binding between Siglecs and recognition ligands was also reported, despite the bivalency of the tested Siglec-Fc, when polyacrylamide probe 
beads with pendant carbohydrate ligands were used for the recognition of Siglecs. ${ }^{36}$ In the research presented here, the multivalent presentation of the Siglecs was achieved by crosslinking the different dimeric Siglec-Fc chimaeric proteins with an anti-Fc-lgG antibody. When the mSiglec-E-Fc/antibody-complex is added to a solution of glyconanoparticles, the $m S i g l e c-E$ in the antibody-complex will recognise the sialic acid on the nanoparticles surface and will bind to it. mSiglec-E has been reported to bind strongly to $\alpha 2,6$ - and $\alpha 2,3-$ linked sialic acids (Fig. S3); 3, 4, 12 thus, it is anticipated that mSiglec-E should recognise the sialic acid ligand 1 present on the designed glyconanoparticles. The binding of the mSiglec-E to the sialic acid will induce the aggregation of the glyconanoparticles (Fig. 2a) and a colour change in the solution should be observed by eye and also by recording the UV-Vis extinction spectrum of the solution before and after addition of the mSiglec-E-Fc/antibody-complex.

The effect of the carbohydrate density on the surface of the AuNPs upon the efficacy of the binding to targets of interest has been highlighted previously. ${ }^{31,34}$ To achieve the optimal plasmonic detection of mSiglec-E, AuNPs were functionalised with different ratios of sialic acid ligand 1:PEG ligand 2, i.e. 100:0, 50:50, 25:75 and 5:95. The ligands were first mixed in the different ratios, ensuring that the total concentration of ligands remained constant, and then added to a solution of citrate-reduced AuNPs. As reported by other authors, the final ratio of ligands functionalising the surface of nanoparticles is highly dependent on the kinetics of the Au-S bond formation for each ligand and thus, on the nature of the ligands used. ${ }^{48}$ Consequently, to control the ratio of the ligands on the surface of the nanoparticles and following the work reported previously by Barrientos et al., ${ }^{49}$ we selected PEG ligand 2 as the dilutant since it is structurally equivalent to the aliphatic side chain of the sialic acid ligand 1. Thus, the sialic acid ligand 1:PEG ligand 2 ratio functionalising the surface of the nanoparticles was expected to be in the same ratio as the molar ratio added to the solution of citrate-reduced AuNPs. To obtain the optimal ligand density on the glyconanoparticles for the plasmonic detection of mSiglec-E, increasing amounts of mSiglec-E-Fc/antibody-complex (from 0 to $4.7 \mu \mathrm{g}$ ) were added to the corresponding glyconanoparticles solution and the UVVis extinction spectrum of each sample was measured before and after addition of the mSiglec-E-Fc/antibody-complex (Fig. 3). The addition of mSiglec-E-Fc/antibody-complex to AuNPs functionalised with sialic acid ligand 1:PEG ligand 2 (100:0) induced only a slight shift in the surface plasmon absorption band (Fig. 3a), suggesting that a full monolayer of sialic 
acid derivative was not a suitable coverage to induce aggregation of the particles in the presence of the mSiglec-E-Fc/antibody-complex. Reducing the sialic acid coverage, AuNPs functionalised with sialic acid ligand 1:PEG ligand 2 (50:50), proved to be a more sensitive assay for the plasmonic detection of mSiglec-E (Fig. 3b). While high concentrations of the Siglec were still required to induce aggregation, there was a greater shift in the surface plasmon absorption band (indicated with a horizontal arrow in Fig. 3b) as compared to the shift observed in Fig. 3a for a full monolayer coverage of the sialic acid ligand. The efficiency in the aggregation of the sialic acid ligand 1:PEG ligand 2 (50:50) in the presence of the mSiglec-E-Fc/antibody-complex was also confirmed by the broadening of the surface plasmon absorption band and the change in extinction intensity at $620 \mathrm{~nm}$ (indicated with a vertical arrow in Fig. 3b) observed upon addition of increasing amounts of the complex. When the sialic acid density on the surface of the nanoparticles was further reduced, sialic acid ligand 1:PEG ligand 2 (25:75) and (5:95), only small changes in the surface plasmon absorption band were observed in the presence of the mSiglec-E-Fc/antibody-complex (Fig. $3 c$ and $3 d$, respectively). It is apparent that when the carbohydrate density on the surface of the nanoparticles is low, the binding interactions between the Siglec and the sialic acid are significantly reduced and, therefore, the aggregation of the AuNPs is less likely to occur. 

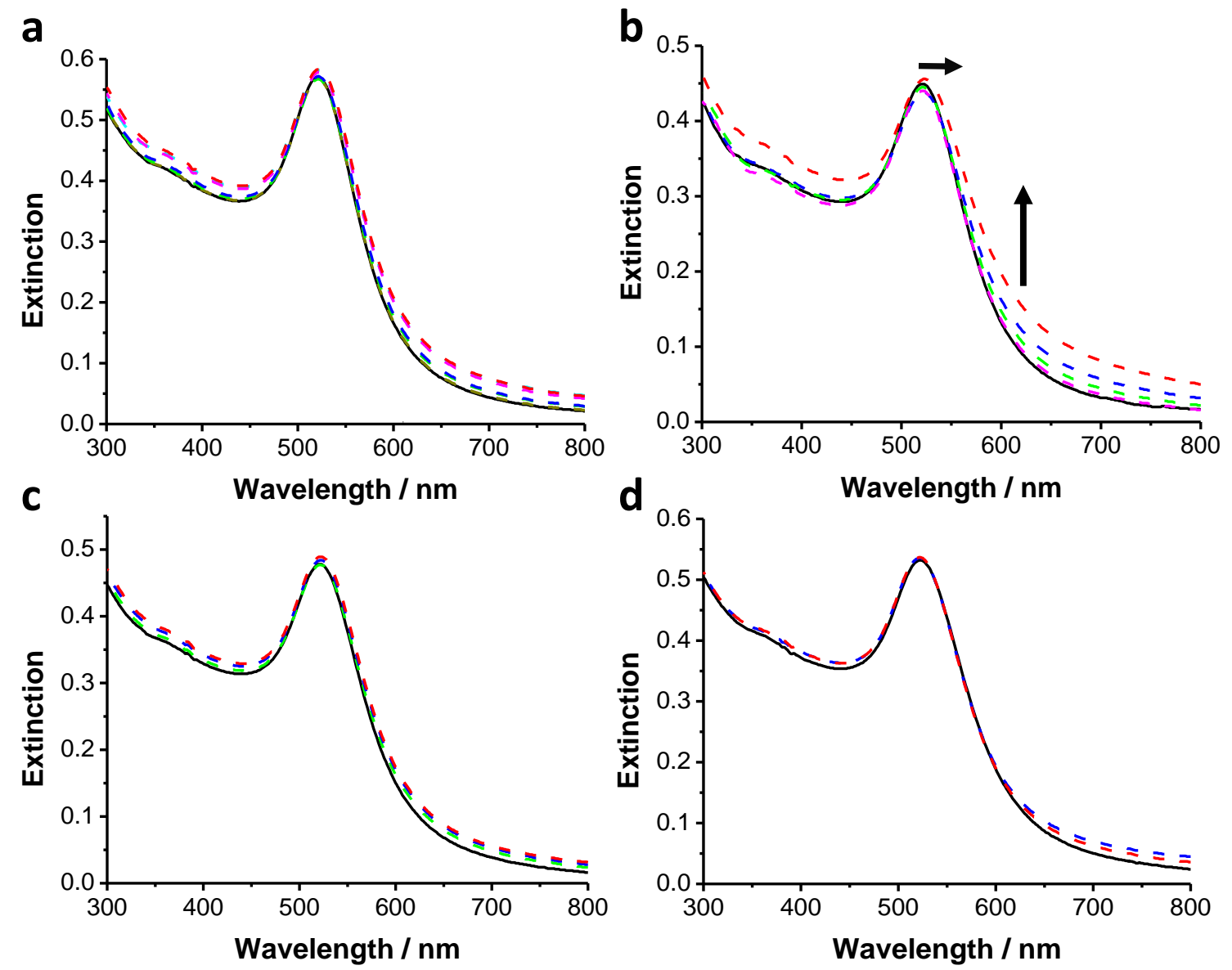

Fig. 3. Optimisation of sialic acid ligand 1:PEG ligand 2 functionalised AuNPs. UV-Vis extinction spectra of different samples of the glyconanoparticles following addition of mSiglec-E-Fc/antibody-complex (from 0 (black) to $4.7 \mu \mathrm{g}$ (red)). The different glyconanoparticles contain sialic acid ligand 1:PEG ligand 2 ratios of: a) 100:0, b) 50:50 (the vertical arrow highlights the increase in extinction intensity at $620 \mathrm{~nm}$ while the horizontal arrow highlights the red-shift in the extinction maximum of the surface plasmon absorption band), c) 25:75 and d) 5:95.

Upon addition of mSiglec-E-Fc/antibody-complex $(4.7 \mu \mathrm{g})$, the greatest change in the extinction intensity at $620 \mathrm{~nm}$ was 0.065 units; while the greatest shift in the wavelength extinction maximum was $3 \mathrm{~nm}$. Both changes were observed for the AuNPs functionalised with sialic acid ligand 1:PEG ligand 2 (50:50) (Fig. 3b). These results suggest that the 50:50 ratio of sialic acid ligand 1:PEG ligand 2 on the AuNPs, glyconanoparticles 1, exhibit the greatest interaction with the $\mathrm{mSiglec}-\mathrm{E}$ inducing the largest aggregation of the glyconanoparticles upon addition of the Fc-fusion protein. 
TEM was used to further confirm the aggregation of the glyconanoparticles 1 in the presence of the mSiglec-E-Fc/antibody-complex. TEM images of the glyconanoparticles 1 before addition of mSiglec-E-Fc/antibody-complex showed dispersed nanoparticles (Fig. 4a and S4a). When the glyconanoparticles 1 were mixed with mSiglec-E-Fc/antibody-complex $(2.35 \mu \mathrm{g})$ for $2 \mathrm{~h}$, networks of particles were observed in the TEM images of the sample, confirming the aggregation of the particles (Fig. $4 \mathrm{~b}$ and S4b). The aggregation of the particles was also observed by the naked eye with a colour change from red to light pink that was visible 10 min following addition of the mSiglec-E-Fc/antibody-complex (Fig. 4c).
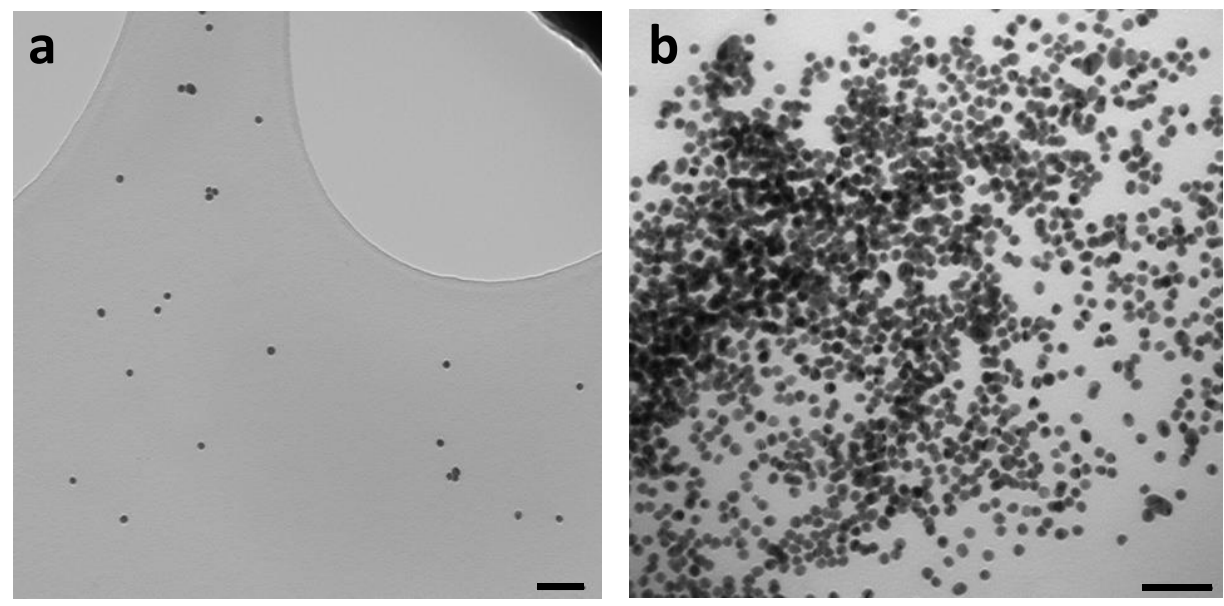

C

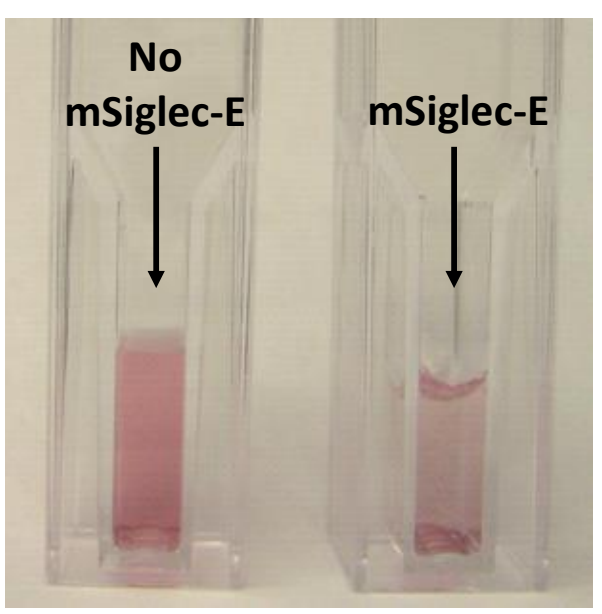

Fig. 4. Transmission electron micrographs of a sample of the glyconanoparticles 1: a) before and b) $2 \mathrm{~h}$ after addition of mSiglec-E-Fc/antibody-complex $(2.35 \mu \mathrm{g})$. The scale bars represent $100 \mathrm{~nm}$. c) Glyconanoparticles 1 (left) before and (right) $10 \mathrm{~min}$ after addition of mSiglec-E-Fc/antibody-complex (2.35 $\mu \mathrm{g})$. 
Two control experiments were performed to confirm that the aggregation of the glyconanoparticles 1 in the presence of mSiglec-E-Fc/antibody-complex was specifically due to the binding of the mSiglec-E to the sialic acids on the glyconanoparticle surface. Increasing amounts of goat anti-human-Fc-antibody (from 0 to $6.25 \mu \mathrm{g}$ ) were added to a solution of glyconanoparticles 1 and the UV-Vis extinction spectrum was recorded following each addition. Changes of the surface plasmon absorption band of the glyconanoparticles 1 upon addition of the control antibody were negligible (Fig. 5a). Thus, the changes observed in the surface plasmon absorption band of the glyconanoparticles in the presence of the mSiglec-E-Fc/antibody-complex (Fig. $3 b$ ) were due to the mSiglec-E. To confirm the importance of the sialic acid for the specific detection of mSiglec-E, AuNPs functionalised with the PEG ligand $\mathbf{2}$ alone were synthesised and the UV-Vis extinction spectrum of the sample was measured before and after addition of increasing amounts of mSiglec-EFc/antibody-complex (Fig. 5b). No changes in the extinction spectrum were observed following addition of the mSiglec-E-Fc/antibody-complex, which further highlights the importance of the sialic acid derivative for the detection of mSiglec-E using the glyconanoparticles.
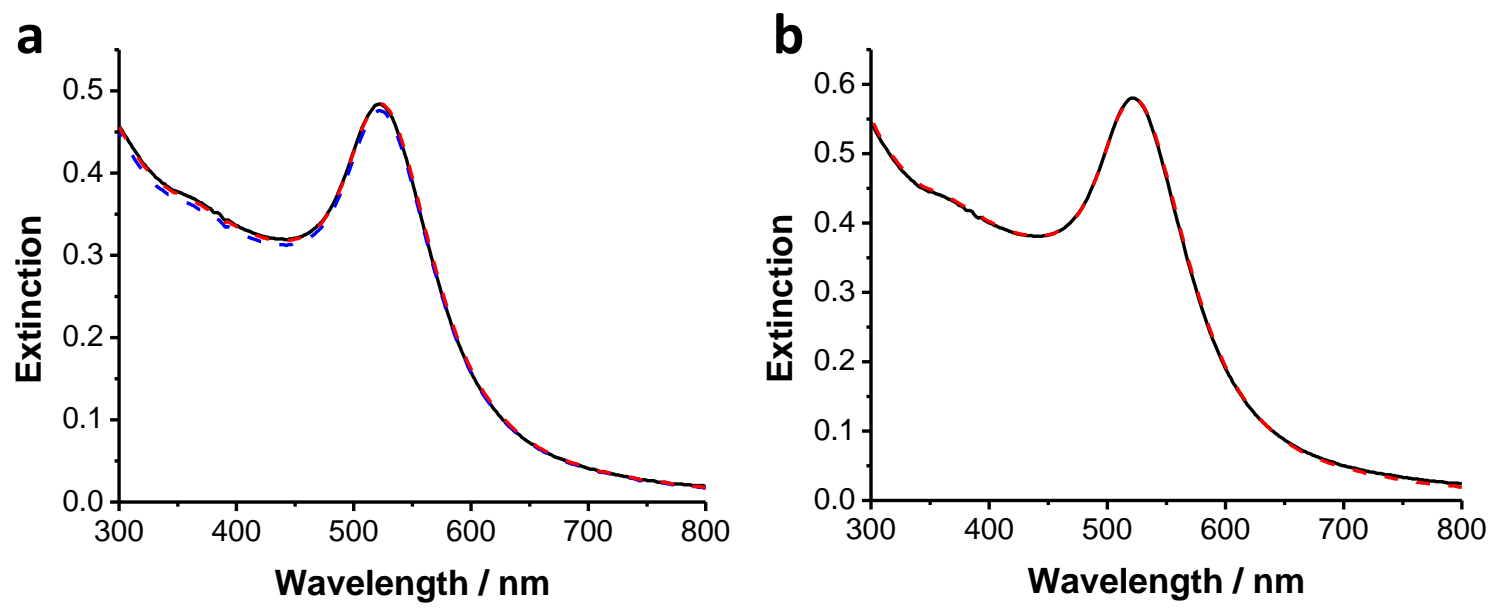

Fig. 5. UV-Vis extinction spectra of: a) glyconanoparticles 1 before (black line) and following addition of goat anti-human-Fc-antibody: $3.12 \mu \mathrm{g}$ (blue line) and $6.25 \mu \mathrm{g}$ (red line); and b) PEG ligand 2 functionalised AuNPs before (black line) and following addition of mSiglec-EFc/antibody-complex (red line, $2.35 \mu \mathrm{g}$ ). 
A further control experiment was performed to investigate the binding affinity of glyconanoparticles 1 towards hSiglec-7. hSiglec-7 expresses a unique preference for $\alpha 2,8$ linked disialic acid over $\alpha 2,6$-linked and $\alpha 2,3$-linked sialic acids (Fig. S3). ${ }^{3,4}$, 12, 50 While both hSiglec-7 and hSiglec-9 are structurally similar to mSiglec-E, the binding specificity of hSiglec-7 is more similar to that of mSiglec-E (Fig. S3). Thus, it was considered that hSiglec-7 would be a suitable control to study the specificity of binding of the glyconanoparticles 1 . No change of the surface plasmon absorption band of glyconanoparticles 1 was observed upon addition of the hSiglec-7-Fc/antibody-complex (2.35 $\mu \mathrm{g}$ ) (Fig. S5). Further, the changes of the surface plasmon absorption band of the glyconanoparticles upon addition of the hSiglec-7-Fc/antibody-complex were compared with those observed upon addition of the same amount of the mSiglec-E-Fc/antibody-complex (Fig. S5). These results confirm that no aggregation of the glyconanoparticles took place in the presence of hSiglec-7-Fc/antibodycomplex. However, a significant aggregation occurred when the mSiglec-E-Fc/antibodycomplex was added, highlighting the specificity of the interaction between the glyconanoparticles 1 and the mSiglec-E-Fc/antibody-complex.

\section{Detection of Siglecs expressed on the surface of transfected CHO cells using the glyconanoparticles 1}

Once the binding affinity of the optimised glyconanoparticles towards mSiglec-E was confirmed in solution, glyconanoparticles $\mathbf{1}$ were used to detect the mSiglec-E expressed on the surface of transfected $\mathrm{CHO}$ cells. The sialic acid on the surface of the glyconanoparticles should recognise and bind to the mSiglec-E expressed on the surface of the cells (as schematically shown in Fig. 2b) which then could be confirmed by TEM. CHO cells expressing mSiglec-E and wild-type CHO cells (without mSiglec-E) were grown, collected for use, centrifuged and resuspended in Tris-buffer. $10^{6}$ of each of the cells were individually added to a solution of the glyconanoparticles $\mathbf{1}$ and the UV-Vis extinction spectrum of the glyconanoparticles was measured before and after addition of the cells. No changes in the surface plasmon absorption band of the glyconanoparticles were observed in the presence of the cells (results not shown). A sample of the glyconanoparticles 1 containing $10^{6} \mathrm{CHO}$ cells expressing mSiglec-E was imaged using TEM (Fig. 6 and S6). The TEM images 
(particularly Fig. 6b) clearly show the glyconanoparticles 1 associated with the periphery of the cell material. The designed glyconanoparticles 1 were bound to the $\mathrm{mSiglec}-\mathrm{E}$ expressed on the surface of the transfected $\mathrm{CHO}$ cells via the sialic acid ligand 1 . These TEM images also explain why aggregation and the related shift in the surface plasmon absorption band were not observed for the solution of the glyconanoparticles in the presence of the $\mathrm{CHO}$ cells. The nanoparticles are not closely packed together, but are dispersed over the cell surface, suggesting that the receptors on the cell membrane are too far apart for the surface plasmon fields of individual nanoparticles to interact with one another. The nanoparticles attached to the cells were found to be $c a .15 \mathrm{~nm}$ in diameter, corresponding to the size of the glyconanoparticles 1. The cells observed in Fig. 6 and S6 were stained using lead citrate to increase the contrast by interacting with proteins and glycogens. When a sample is stained with lead citrate and in the presence of carbon dioxide, a precipitate due to the formation of lead carbonate could potentially contaminate the sample and could be seen as 'nanoparticles' using TEM. To confirm that the $15 \mathrm{~nm}$ particles observed in Fig. 6 and S6 were the bound glyconanoparticles, a sample of glyconanoparticles 1 in the presence $10^{6}$ $\mathrm{CHO}$ cells expressing mSiglec-E was imaged in the TEM without staining (Fig. S7). The TEM images show that the glyconanoparticles are still present and again appear at the periphery of the cells. 

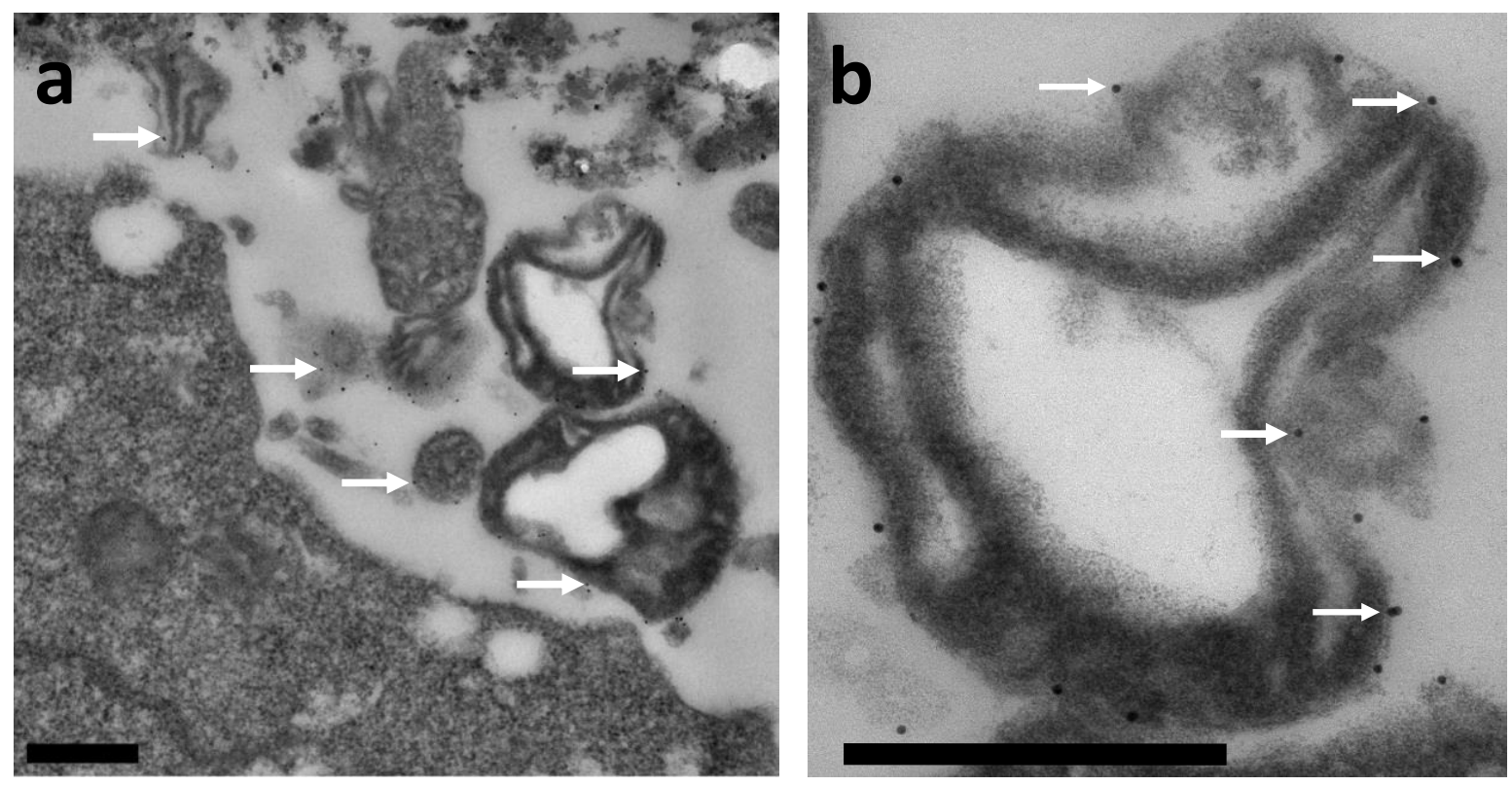

Fig. 6. TEM images of stained samples of $\mathrm{CHO}$ cells expressing mSiglec-E in the presence of glyconanoparticles 1: a) scale bar: $500 \mathrm{~nm}$, magnification: 7500x; and b) focused image of a selected area of a), scale bar: $500 \mathrm{~nm}$. White arrows highlight five of the glyconanoparticles 1 bound to the cell surface.

To ensure that the glyconanoparticles are specifically binding to the $\mathrm{mSiglec}-\mathrm{E}$ receptors on the cell surface, wild-type $\mathrm{CHO}$ cells not expressing mSiglec-E on their surface were added to the glyconanoparticles 1 and the sample imaged using TEM (Fig. S8). The TEM images show that there are no nanoparticles bound to the surface of the cells confirming that the glyconanoparticles specifically bind to the $\mathrm{CHO}$ cells expressing mSiglec-E via sialic acidSiglec interactions. In a final control experiment, mSiglec-E CHO cells without nanoparticles were imaged following staining of the sample (Fig. S9). As expected, no nanoparticles were observed in the TEM images of the control sample.

\section{Conclusions}

The primary aim of this research was to detect the sialic acid-binding immunoglobulin-like lectin mSiglec-E overexpressed on the surface of Chinese hamster ovary cells. Siglecs play a wide range of biological roles regulating functions in the immune and nervous systems and being markers of disease stages including cancer. Methods for the detection of cellular 
Siglecs should be able to overcome problems such as cis interactions and low Siglec-ligand affinity. Bioanalytical detection tools based on nanosystems can overcome these problems since they result in multivalent ligand-based probes. While several studies have been recently reported describing the use of nanoparticle-based systems to target Siglecs, ${ }^{21-24}$ the fluorescence-based techniques previously used do not provide information regarding the localisation of single nanoparticles on the cellular surface. Here we describe a system that enables single particle detection and thus, assessment of Siglec distribution on the cell surface, using sialic acid functionalised gold nanoparticles in combination with transmission electron microscopy. Gold nanoparticles (ca. $15 \mathrm{~nm}$ ) were functionalised with different ratios of a sialic acid derivative ligand $\mathbf{1}$, diluted with PEG ligand $\mathbf{2}$, which is able to specifically recognise mSiglec-E. The optimum sialic acid density on the surface of the nanoparticles for the detection of mSiglec-E was investigated in solution via the plasmonic detection of mSiglec-E-Fc/antibody-complex. The optimised glyconanoparticles for the detection of the soluble mSiglec-E were found to be those containing sialic acid ligand $\mathbf{1}$ and PEG ligand 2 in a 50:50 ratio, glyconanoparticles 1 . The addition of the mSiglec-EFc/antibody-complex to a solution of glyconanoparticles $\mathbf{1}$ induced the aggregation of the nanoparticles which was observed by a shift of the surface plasmon absorption band, a change in the colour of the solution 10 min following addition of the complex and also by imaging of the sample using TEM. The changes in the surface plasmon absorption band of the optimised glyconanoparticles were not observed in the presence of an anti-Fc antibody only or in the presence of the structurally similar hSiglec-7-Fc/antibody-complex, confirming the specificity of the glyconanoparticles 1 towards mSiglec-E. The importance of the sialic acid for the specific plasmonic detection of mSiglec-E was confirmed when the addition of mSiglec-E-Fc/antibody-complex to a solution of AuNPs functionalised with only PEG ligand 2 produced negligible changes in the surface plasmon absorption band of the sample. The optimised glyconanoparticles 1 were then used for the detection of mSiglec-E expressed on the surface of transfected $\mathrm{CHO}$ cells using TEM. TEM enabled the visualisation of single glyconanoparticles over the surface of the imaged cells. Binding of the nanoparticles to the cell surface was not observed when wild-type $\mathrm{CHO}$ cells, without the mSiglec-E, were treated with the glyconanoparticles 1 . The bioassay described provides an easy to perform methodology to assess the specific recognition of ligands by the respective sialic acidbinding immunoglobulin-like lectin Siglec and with the same glyconanoparticle platform to 
visualise, with high resolution, the distribution of Siglecs on the surface of a cell using TEM. The developed method can be used in the future to assess the distribution of Siglecs expressed on the surface of different cell types and can consequently, contribute to further our understanding of the biological roles that these lectins play in vivo.

\section{Acknowledgements}

These studies were supported by Basic Technology Grant GR/S79268/02 from Research Councils UK, the UK BBSRC Institute Strategic Programme Grant on Understanding and Exploiting Metabolism (MET) [BB/J004561/1], the EPSRC (Grant GR/S64134/01) and the John Innes Foundation. MJM thanks the School of Chemistry, University of East Anglia for financial support. The authors are grateful to Rick Evans-Gowing (University of East Anglia) for assistance with the TEM images.

\section{References}

1. P. R. Crocker and A. Varki, Immunology, 2001, 103, 137-145.

2. P. R. Crocker, Curr. Opin. Pharmacol., 2005, 5, 431-437.

3. P. R. Crocker, J. C. Paulson and A. Varki, Nat. Rev. Immunol., 2007, 7, 255-266.

4. M. S. Macauley, P. R. Crocker and J. C. Paulson, Nat. Rev. Immunol., 2014, 14, 653-666.

5. S. Von Gunten and B. S. Bochner, Ann. N. Y. Acad. Sci., 2008, 1143, 61-82.

6. F. Schwarz, J. Fong and A. Varki, in Biochemical Roles of Eukaryotic Cell Surface Macromolecules, eds. A. Chakrabarti and A. Surolia, Springer International Publishing, 2015, vol. 842, ch. 1, pp. 1-16.

7. C. Vitale, C. Romagnani, M. Falco, M. Ponte, M. Vitale, A. Moretta, A. Bacigalupo, L. Moretta and M. C. Mingari, Proc. Natl. Acad. Sci. U. S. A., 1999, 96, 15091-15096.

8. C. Vitale, C. Romagnani, A. Puccetti, D. Olive, R. Costello, L. Chiossone, A. Pitto, A. Bacigalupo, L. Moretta and M. C. Mingari, Proc. Natl. Acad. Sci. U. S. A., 2001, 98, 57645769.

9. K. Cheent and S. I. Khakoo, Immunology, 2009, 126, 449-457.

10. T. Angata and A. Varki, J. Biol. Chem., 2000, 275, 22127-22135.

11. J. Q. Zhang, G. Nicoll, C. Jones and P. R. Crocker, J. Biol. Chem., 2000, 275, 22121-22126.

12. J. Q. Zhang, B. Biedermann, L. Nitschke and P. R. Crocker, Eur. J. Immunol., 2004, 34, 1175-1184. 
13. P. R. Crocker and J. Q. Zhang, in Glycogenomics: The Impact of Genomics and Informatics on Glycobiology, eds. K. Drickamer and A. Dell, 2002, pp. 83-94.

14. Z. Yu, M. Maoui, L. Wu, D. Banville and S. Shen, Biochem. J., 2001, 353, 483-492.

15. M. Zhang, T. Angata, J. Y. Cho, M. Miller, D. H. Broide and A. Varki, Blood, 2007, 109, 4280-4287.

16. M. K. O'Reilly and J. C. Paulson, Trends Pharmacol. Sci., 2009, 30, 240-248.

17. A. D. Ricart, Clin. Cancer Res., 2011, 17, 6417-6427.

18. V. H. J. van der Velden, J. G. te Mervelde, P. G. Hoogeveen, I. D. Bernstein, A. B. Houtsmuller, M. S. Berger and J. J. M. van Dongen, Blood, 2001, 97, 3197-3204.

19. C. Jandus, K. F. Boligan, O. Chijioke, H. Liu, M. Dahlhaus, T. Démoulins, C. Schneider, M. Wehrli, R. E. Hunger, G. M. Baerlocher, H.-U. Simon, P. Romero, C. Münz and S. von Gunten, J. Clin. Invest., 2014, 124, 1810-1820.

20. C. Scott, W. Marouf, D. Quinn, R. Buick, S. Orr, R. Donnelly and P. McCarron, Pharm. Res., 2008, 25, 135-146.

21. C. D. Rillahan, M. S. Macauley, E. Schwartz, Y. He, R. McBride, B. M. Arlian, J. Rangarajan, V. V. Fokin and J. C. Paulson, Chem. Sci., 2014, 5, 2398-2406.

22. N. Kawasaki, J. L. Vela, C. M. Nycholat, C. Rademacher, A. Khurana, N. van Rooijen, P. R. Crocker, M. Kronenberg and J. C. Paulson, Proc. Natl. Acad. Sci. U. S. A., 2013, 110, 7826-7831.

23. C. D. Rillahan, E. Schwartz, R. McBride, V. V. Fokin and J. C. Paulson, Angew. Chem., Int. Ed., 2012, 51, 11014-11018.

24. C. D. Rillahan, E. Schwartz, C. Rademacher, R. McBride, J. Rangarajan, V. V. Fokin and J. C. Paulson, ACS Chem. Biol., 2013, 8, 1417-1422.

25. X. Yu, A. Feizpour, N.-G. P. Ramirez, L. Wu, H. Akiyama, F. Xu, S. Gummuluru and B. M. Reinhard, Nat. Commun., 2014, 5, Article number 4136.

26. Y. Sun and Y. Xia, Analyst, 2003, 128, 686-691.

27. M. J. Marín, C. L. Schofield, R. A. Field and D. A. Russell, Analyst, 2015, 140, 59-70.

28. D. C. Hone, A. H. Haines and D. A. Russell, Langmuir, 2003, 19, 7141-7144.

29. R. Karamanska, B. Mukhopadhyay, D. A. Russell and R. A. Field, Chem. Commun., 2005, 3334-3336.

30. C. L. Schofield, R. A. Field and D. A. Russell, Anal. Chem., 2007, 79, 1356-1361.

31. C. L. Schofield, B. Mukhopadhyay, S. M. Hardy, M. B. McDonnell, R. A. Field and D. A. Russell, Analyst, 2008, 133, 626-634.

32. C. Lee, M. A. Gaston, A. A. Weiss and P. Zhang, Biosens. Bioelectron., 2013, 42, 236-241.

33. I. Papp, C. Sieben, K. Ludwig, M. Roskamp, C. Bottcher, S. Schlecht, A. Herrmann and R. Haag, Small, 2010, 6, 2900-2906.

34. M. J. Marín, A. Rashid, M. Rejzek, S. A. Fairhurst, S. A. Wharton, S. R. Martin, J. W. McCauley, T. Wileman, R. A. Field and D. A. Russell, Org. Biomol. Chem., 2013, 11, 71017107. 
35. J. Simpson, D. Craig, K. Faulds and D. Graham, Nanoscale Horiz., 2016, 1, 60-63.

36. M. K. O’Reilly and J. C. Paulson, Methods Enzymol., 2010, 478, 343-363.

37. A. Varki, Siglecs. In: Encyclopedia of Biological Chemistry, Elsevier, Oxford, 2nd edn., 2013.

38. A. Varki and T. Angata, Glycobiology, 2006, 16, 1R-27R.

39. S. M. Khersonsky, D.-W. Jung, T.-W. Kang, D. P. Walsh, H.-S. Moon, H. Jo, E. M. Jacobson, V. Shetty, T. A. Neubert and Y.-T. Chang, J. Am. Chem. Soc., 2003, 125, 1180411805.

40. B. V. Enüstün and J. Turkevich, J. Am. Chem. Soc., 1963, 85, 3317-3328.

41. R. Karamanska, J. Clarke, O. Blixt, J. MacRae, J. Zhang, P. R. Crocker, N. Laurent, A. Wright, S. Flitsch, D. A. Russell and R. A. Field, Glycoconj. J., 2008, 25, 69-74.

42. G. Nicoll, J. Ni, D. Liu, P. Klenerman, J. Munday, S. Dubock, M.-G. Mattei and P. R. Crocker, J. Biol. Chem., 1999, 274, 34089-34095.

43. H. O. Ok, J. L. Szumiloski, G. A. Doldouras, W. R. Schoen, K. Cheng, W. W. S. Chan, B. S. Butler, R. G. Smith, M. H. Fisher and M. J. Wyvratt, Bioorg. Med. Chem. Lett., 1996, 6, 3051-3056.

44. O. Blixt and T. Norberg, J. Org. Chem., 1998, 63, 2705-2710.

45. C. Jones, M. Virji and P. R. Crocker, Mol. Microbiol., 2003, 49, 1213-1225.

46. T. Avril, E. R. Wagner, H. J. Willison and P. R. Crocker, Infect. Immun., 2006, 74, 41334141.

47. M. A. Campanero-Rhodes, R. A. Childs, M. Kiso, S. Komba, C. Le Narvor, J. Warren, D. Otto, P. R. Crocker and T. Feizi, Biochem. Biophys. Res. Commun., 2006, 344, 1141-1146.

48. M. Marradi, F. Chiodo, I. Garcia and S. Penadés, Chem. Soc. Rev., 2013, 42, 4728-4745.

49. Á. G. Barrientos, J. M. de la Fuente, T. C. Rojas, A. Fernández and S. Penadés, Chem. Eur. J., 2003, 9, 1909-1921.

50. T. Yamaji, T. Teranishi, M. S. Alphey, P. R. Crocker and Y. Hashimoto, J. Biol. Chem., 2002, 277, 6324-6332. 